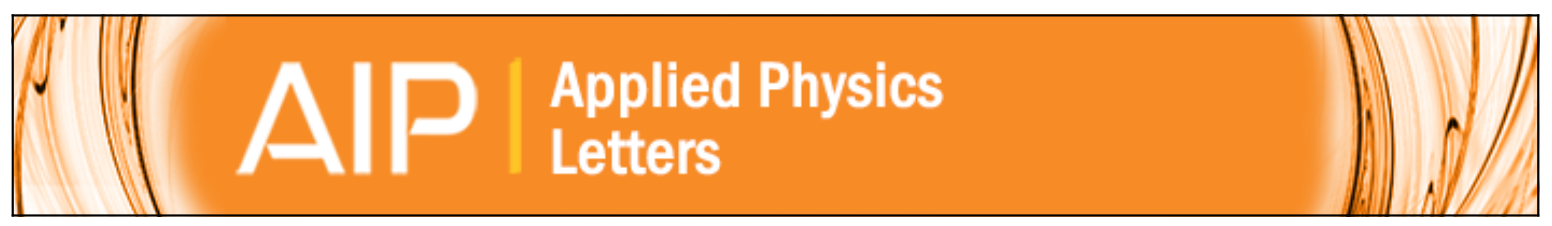

\title{
Modeling the lasing spectra of InAs/InP Quantum dash lasers
}

M. Z. M. Khan, T. K. Ng, U. Schwingenschlogl, P. Bhattacharya, and B. S. Ooi

Citation: Applied Physics Letters 98, 101105 (2011); doi: 10.1063/1.3560052

View online: http://dx.doi.org/10.1063/1.3560052

View Table of Contents: http://scitation.aip.org/content/aip/journal/apl/98/10?ver=pdfcov

Published by the AIP Publishing 


\title{
Modeling the lasing spectra of InAs/InP Quantum dash lasers
}

\author{
M. Z. M. Khan, ${ }^{1}$ T. K. Ng, ${ }^{1}$ U. Schwingenschlogl, ${ }^{1}$ P. Bhattacharya, ${ }^{2}$ and B. S. Ooi ${ }^{1, a)}$ \\ ${ }^{1}$ Division of Physical Sciences and Engineering, King Abdullah University of Science and Technology \\ (KAUST), Thuwal 23955-6900, Saudi Arabia \\ ${ }^{2}$ Department of Electrical Engineering and Computer Science, University of Michigan, 1301, Beal Avenue, \\ Ann Arbor, Michigan 48109-2122, USA
}

(Received 4 January 2011; accepted 7 February 2011; published online 9 March 2011)

\begin{abstract}
We report a theoretical model for InAs/InP quantum-dash (Qdash) lasers incorporating a coupled set of rate equations taking into account the inhomogeneous broadening due to Qdash size fluctuation, the homogeneous broadening due to optical gain of a single Qdash, and the longitudinal cavity modes. The role of cavity length on the Qdash lasing characteristics, particularly the redshift in the peak lasing wavelength, is analyzed and compared with the experimental results by attributing it to the active region inhomogeneity. (C) 2011 American Institute of Physics.
\end{abstract}

[doi:10.1063/1.3560052]

InAs/InP quantum-dash (Qdash) lasers performance has shown tremendous improvements, particularly the broadband emission spanning $\sim 85 \mathrm{~nm}$, in the last decade because of its potential application in long haul optical communication. ${ }^{1-5}$ This may be attributed to the near singular density of state (DOS), experimental optimization of design/fabrication process parameters, ${ }^{1-8}$ and intensive theoretical research. ${ }^{9-15}$ Various numerical techniques have been developed to address the Qdash materials and devices, but mostly concentrating on the energy band and emission characteristics of Qdash structures ${ }^{10-14}$ rather than studying the Qdash devices except for a few which looked into Qdash semiconductor optical amplifier $(\mathrm{SOA})^{15}$ and dynamic characteristics of Qdash laser. ${ }^{16}$ Recently, Tan et $a l^{6}{ }^{6}$ and Zhou et al. ${ }^{7}$ has experimentally reported that increasing the cavity length result in a redshift of the lasing wavelength from the InAs/InP Qdash lasers which they attribute to the unique DOS of the Qdashes. Inspite of this unusual behavior, no research has been done to confirm this observation theoretically and find out the reason behind it. This may probably be due to a lack of most of the reported models to analyze the lasing emission spectra of Qdash lasers.

In this letter, we report a theoretical model that can be utilized to study both the lasing emission and dynamical characteristics of InAs/InP Qdash lasers, in general. Based on our calculations, we confirm the redshift in the lasing wavelength and consider an energy band model to qualitatively explain the reason behind this observation.

Our model is based on a set of coupled rate equations and the density matrix formulation incorporating the unique features of the Qdash DOS and the gain characteristics similar to the work reported by Sugawara et al. ${ }^{9}$ for quantum dot (Qdot) lasers and Hadaas et al. ${ }^{15}$ for Qdash SOA. Figure 1 illustrates the energy band diagram of our model. The figure shows the energy states of the laser active medium and the relaxation (re-excitation) process of carriers into the Qdash ground state (GS). We assume that only a single GS is formed inside the Qdash, which is characterized by the DOS function, and that charge neutrality always holds in each Qdash. The injected carriers diffuse through the separate

${ }^{\text {a)} E l e c t r o n i c ~ m a i l: ~ b o o n . o o i @ k a u s t . e d u . s a . ~}$ confinement heterostructure (SCH) layer, relax (capture) into the wetting layer (WL), and then relax into the dashes GS. The respective time constants are shown in the figure. In addition, further assumptions are made in the model according to the reported Qdot model. ${ }^{9}$ The dashes are grouped according to their resonant wavelength and a series of longitudinal photon modes is considered over the interband transition energy. The model considers $2 M_{d}+1$ dash groups with $N$ intradash energy levels in each group and $2 M_{p}+1$ photon modes $\left(M_{d}\right.$ dash group corresponds to the group of central transition energy $\left.E_{c v}\right)$. The GS energy level of a Qdash group is $E_{j, 0}$, with $E_{j, N}$ being the highest energy of that group, and $E_{j, k}$ is a generic energy level of the system where $j(m)$ $=0,1, \ldots, 2 M_{d}\left(2 M_{p}\right)$ and $k=0,1, \ldots, N$. The difference in resonant energy between adjacent dash groups is $\Delta E_{j}$ and the intradash energy difference is $\Delta E_{k}{ }^{9,15}$ The relative DOS weight of Qdash groups is determined by the envelope of a Gaussian inhomogeneous broadening (GIB) function. The model is as follows:

$$
d N_{S} / d t=\eta_{i} I / e-N_{S} / \tau_{S W}-N_{S} / \tau_{S}+N_{W} / \tau_{W S}
$$

$$
\begin{aligned}
d N_{W} / d t= & N_{S} / \tau_{S W}+\sum_{j, k} N_{j, k} / \tau_{D W}^{j, k}-N_{W} / \overline{\tau_{W D}}-N_{W} / \tau_{W S} \\
& -N_{W} / \tau_{W},
\end{aligned}
$$

$$
\begin{aligned}
d N_{j, k} / d t= & N_{W} G_{j, k} / \tau_{W D}^{j, k}-N_{j, k} / \tau_{D W}^{j, k}-N_{W} / \tau_{D} \\
& -\left(c \Gamma / n_{a}\right) \sum_{m} g_{m}^{j, k} S_{m},
\end{aligned}
$$

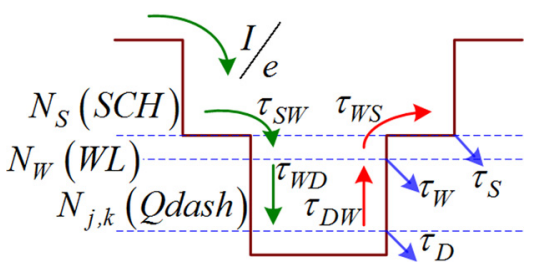

FIG. 1. (Color online) Energy band diagram of the conduction band of the Qdash laser including the SCH, WL, and GS energy levels. 
TABLE I. Qdash laser parameters used in the model.

\begin{tabular}{|c|c|c|c|}
\hline Parameter & Description & Value & Unit \\
\hline$d$ & Stripe width & $40^{\mathrm{a}}$ & $\mu \mathrm{m}$ \\
\hline$w_{W L}$ & WL thickness & 1 & $\mathrm{~nm}$ \\
\hline$w_{D h}$ & Qdash width & $20^{\mathrm{a}}$ & $\mathrm{nm}$ \\
\hline$h_{D h}$ & Qdash height & $1.5^{\mathrm{a}}$ & $\mathrm{nm}$ \\
\hline$N_{l y r}$ & Number of Qdash layers & $4^{\mathrm{a}}$ & $\cdots$ \\
\hline$A_{e f f}$ & Qdash effective crossectional area & $1.0 \times 10^{-12}$ & $\mathrm{~cm}^{2}$ \\
\hline$\eta_{i}$ & Injection efficiency & 1 & $\cdots$ \\
\hline$m_{e}^{*}$ & Electron effective mass & $0.04 m_{0}$ & $\cdots$ \\
\hline$\Gamma$ & Confinement factor & 0.03 & $\cdots$ \\
\hline$R_{1}=R_{2}$ & Cleaved facet reflectivity & 0.3 & $\cdots$ \\
\hline$\alpha_{i}$ & Internal modal loss & $10^{\mathrm{a}}$ & $\mathrm{cm}^{-1}$ \\
\hline$\beta$ & Spontaneous emission factor & $1 \times 10^{-4} \mathrm{~b}$ & $\cdots$ \\
\hline$N_{D}$ & Qdash DOS & $5 \times 10^{17 a}$ & $\mathrm{~cm}^{-3}$ \\
\hline$D_{G}$ & Qdash GS degeneracy & $1^{\mathrm{a}}$ & $\cdots$ \\
\hline$D_{W}$ & WL DOS & $1.8 \times 10^{19} \mathrm{a}$ & $\mathrm{cm}^{-3}$ \\
\hline$E_{C V}$ & Central transition energy & $805^{\mathrm{a}}$ & $\mathrm{meV}$ \\
\hline$E_{W L}$ & WL GS energy & $916^{\mathrm{a}}$ & $\mathrm{meV}$ \\
\hline$\hbar \Gamma_{h o m}$ & Homogeneous broadening & 10 & $\mathrm{meV}$ \\
\hline$\Gamma_{i n h}$ & Inhomogeneous broadening & 25 & $\mathrm{meV}$ \\
\hline$\tau_{S W}$ & Relaxation time from $\mathrm{SCH}$ to $\mathrm{WL}$ & 500 & ps \\
\hline$\tau_{W S}$ & Re-excitation time from WL to $\mathrm{SCH}$ & 1 & ns \\
\hline$\tau_{W D 0}$ & Initial capture time from WL to GS & $2^{\mathrm{a}}$ & ps \\
\hline$\tau_{W}$ & Recombination lifetime of WL & $0.8^{\mathrm{a}}$ & ns \\
\hline$\tau_{D}$ & Recombination lifetime of Qdash & $0.5^{\mathrm{a}}$ & ns \\
\hline$\tau_{S p}$ & Spontaneous lifetime & $2.8^{\mathrm{b}}$ & ns \\
\hline$n_{a}$ & Refractive index of active region & 3.5 & $\cdots$ \\
\hline
\end{tabular}

${ }^{\mathrm{a}}$ See Refs. 8 and 15 .

${ }^{\mathrm{b}}$ See Ref. 9.

$$
\begin{aligned}
d S_{m} / d t= & \beta \sum_{j, k} B\left(E_{m}-E_{j, k}\right) N_{j, k} / \tau_{S p}+\left(c \Gamma / n_{a}\right) \sum_{k, j} g_{m}^{j, k} S_{m} \\
& -S_{m} / \tau_{p},
\end{aligned}
$$

where $N_{S}, N_{W}$, and $N_{j, k}$ are the carrier numbers in the $\mathrm{SCH}$, WL, and the Qdash $E_{j, k}$ level, respectively. Equations (1) and (2) refer to the dynamics governing the carrier population in the SCH and WL, respectively; Eq. (3) corresponds to the carrier dynamics in each of the intradash energy levels of each Qdash group. I corresponds to the current injection, $\overline{\tau_{W D}}$ is the average carrier capture time from WL to the Qdash GS energy level and $\tau_{D W}^{j, k}$ corresponds to the carrier re-excitation (escape) time from the Qdash $E_{j, k}$ level to the WL. ${ }^{9,15} \tau_{W D_{0}}$ is the initial capture time when the $k$ th state of $j$ th Qdash group is unoccupied. $V_{A}=0.75 h_{D h} d L N_{l y r}$ and $V_{W}$ $=w_{W L} d L N_{l y r}$, respectively, are the volume of the active region and the WL and $L$ is the cavity length. The carrier escape rate from the Qdash GS to the WL can be calculated using a detailed balance relationship between carrier escape and carrier capture. ${ }^{15}$

$$
\begin{aligned}
G_{j, k}= & \left(\sqrt{2 \pi} \xi_{0}\right)^{-1} \exp \left[\left(E_{j, 0}-E_{c v}\right)^{2} / 2 \xi_{0}^{2}\right] d E_{j}\left(\sqrt{E_{j, k+1}-E_{j, 0}}\right. \\
& \left.-\sqrt{E_{j, k}-E_{j, 0}}\right) / \sqrt{E_{j, N+1}-E_{j, 0}}
\end{aligned}
$$

is the weighted energy state of the $E_{j, k}$ Qdash group with the right hand side part representing the GIB term with full width at half maximum (FWHM) $\Gamma_{i n h}=2.35 \xi_{0}$ and the ratio of two integrals whose details can be found elsewhere. ${ }^{15}$ Note that $G_{j, k}$ is normalized as $\sum_{j} \Sigma_{k} G_{j, k}=1 . \quad P_{j, k}$ $=N_{j, k} / 2 D_{g} N_{D} V_{k} G_{j, k}$ is the occupational probability (includ-
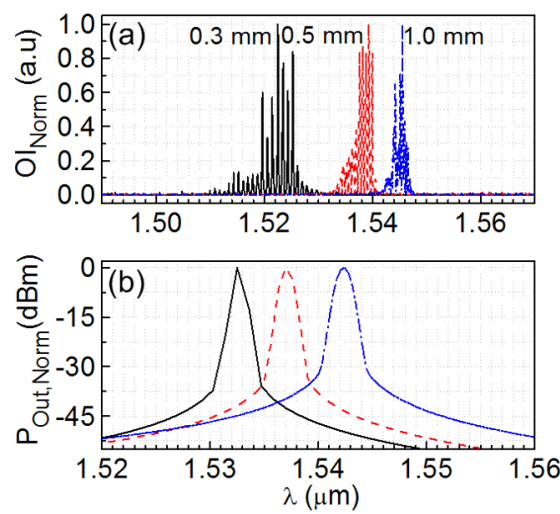

FIG. 2. (Color online) Comparison of the lasing spectra between (a) the experimental data and (b) the calculated results corresponding to the Qdash lasers of (Ref. 6) and (Ref. 8), respectively, at $1.1 J_{\text {th }}$ and different cavity lengths $L=0.3,0.5$, and $1.0 \mathrm{~mm}$. $O I_{\text {norm }}$ and $P_{\text {out,norm }}$ correspond to the normalized optical intensity and normalized calculated output power.

ing the spin), where $N_{D}=A_{e f f}^{-1} \sqrt{2 m_{e}^{*} / \pi^{2} \hbar^{2}} \sqrt{E_{j, N+1}-E_{j, 0}}$ is the Qdash DOS function. ${ }^{15}$ It is worth mentioning that $N_{D}$ and $G_{j, k}$ includes the unique features of the Qdash gain medium.

The multimode photon rate equation of the $m$ th mode is represented by Eq. (4), where $S_{m}$ is the photon population. The first and the last term on the right hand side is the spontaneous emission term and the photon loss, respectively. The photon life time in the cavity is given by $\tau_{p}^{-1}=\left(c / n_{a}\right)\left[\alpha_{i}\right.$ $\left.+\ln \left(1 / R_{1} R_{2}\right) / 2 L\right]$. The second term on the right hand side corresponds to the stimulated emission term where the linear optical gain, derived from the density matrix equation, is given by ${ }^{9-11}$

$$
g_{m}^{j, k}=\frac{2 \pi e^{2} \hbar N_{D}}{c n_{a} \epsilon_{0} m_{0}^{2}} \frac{\left|M_{c v}\right|^{2}}{E_{c v}}\left(2 P_{j, k}-1\right) G_{j, k} B\left(E_{m}-E_{j, k}\right),
$$

where $B\left(E_{m}-E_{j, k}\right)$ is the Lorentzian homogeneous broadening term ${ }^{9,10}$ with FWHM $\hbar \Gamma_{h o m} .\left|M_{c v}\right|^{2}$ is the transition matrix element ${ }^{9,10}$ and $m_{0}$ is the free electron mass. Using the set of above formulae, the steady state laser output power can be calculated by $I_{m}=\hbar \omega_{m} c S_{m} \ln (1 / R) /\left(2 L n_{a}\right)$, where $\hbar \omega_{m}$ is the energy of the $m$ th photon mode. All the other symbols appearing in the model are described in Table I with their respective values, ${ }^{8,9,15} \Delta E_{j}=0.354 \mathrm{meV}, \Delta E_{m}$ $=c h / 2 n_{a} L$ and $M_{d}=200$.

Figure 2(a) shows the room temperature lasing spectra of an InAs/InP Qdash laser structure observed experimentally as a function of cavity length $(L),{ }^{6}$ while Fig. 2(b) shows the central lasing spectra (calculated by identifying the central wavelength at the FWHM of the lasing spectra) of a similar InAs/InP Qdash laser ${ }^{8}$ structure calculated by our model. The injection current is $1.1 J_{t h}$ in both cases. The redshift in the central lasing wavelength at different $L$, as predict by the model, is in good agreement with the experimental data, thus, showing the effectiveness of the model. Note that the lasing wavelength at different $L$ in Figs. 2(a) and 2(b) are relatively different. For instance, considering $L=0.3 \mathrm{~mm}$, the experimental data show a central lasing wavelength of $\sim 1522.6 \mathrm{~nm}$, while the calculated result is $\sim 1532.5 \mathrm{~nm}$. This is expected because the two laser structures are different in terms of growth and fabrication. Therefore, the average size of the dashes, particularly, the height is different. Our objective here is to predict the spectral characteristics of commonly grown Qdash lasers and not to model accurately 


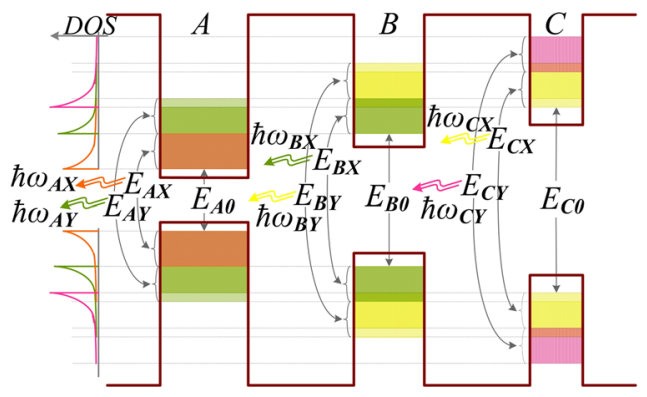

FIG. 3. (Color online) An energy band model sketch describing the process of the red shifting of the central lasing wavelength on increasing the active region inhomogeneity of the Qdash laser.

the energy states of the Qdashes. Therefore, we utilized the staircase approximation to calculate the high energy tail of DOS $N_{D}$ of Qdashes according to Eq. (5). The total redshift observed experimentally ${ }^{6}$ by increasing $L$ from 0.3 to 1.0 $\mathrm{mm}$ is $\sim 20 \mathrm{~nm}$, while the calculated results show a redshift of $\sim 10 \mathrm{~nm}$. However, in the case of (Ref. 7), the observed redshift constitutes more than $40 \mathrm{~nm}$ which may again be attributed to a different active region of the laser structure. Moreover, we have observed that increasing the inhomogeneity from 25 to $45 \mathrm{meV}$ has enhanced the total redshift phenomenon. A value of $\sim 5.5 \mathrm{~nm}$ between 0.5 and $1.0 \mathrm{~mm}$ cavity length has increased to $\sim 11 \mathrm{~nm}$ which is almost double. The reason behind this peculiar observation may be related to the large change in threshold current density as we reduce $L$ and also strongly to the inhomogeneity of the active region which probably increases implicitly due to larger active region volume as a result of longer cavity.

Figure 3 gives a sketch of the energy band model to explain this behavior qualitatively and in a comprehensive manner. The model consists of an energy band diagram of three arbitrary groups of Qdashes labeled A, B, and C with respective GS energies $E_{A 0}, E_{B 0}$, and $E_{C 0}$. For simplicity, the model assumes transition only to the heavy holes. Consider $\mathrm{C}$ to be the central peak lasing wavelength Qdash group at low current injection $\left(1.1 J_{t h}\right)$. Now, if the inhomogeneity of the laser active medium increases (either implicit increase due to cavity length or explicit increase), another two Qdash groups (A and B) may be assumed with GS energies obeying $E_{A 0}<E_{B 0}<E_{C 0}$. The Qdash groups with transition energies $>E_{C 0}$ are redundant as all generated photons in those groups will probably be absorbed by the groups with lower transition energy $<E_{C 0}$. It is worth mentioning that the probability of carriers occupying any energy region of Qdash energy states increases because of an overlapping DOS, particularly in the high energy regions, owing to the spread of energy levels in Qdashes caused by large size fluctuations (i.e., increase in inhomogeneous broadening). The photons generated from the carrier capture in the high energy band (represented by $E_{C Y}$ ) of C, bearing energy $\hbar \omega_{C Y}$ may witness lower transition energy gap $E_{A Y}, E_{B X}$, and $E_{B Y}$ corresponding to A and $\mathrm{B}$. Therefore, the photons (with energy $\hbar \omega_{C Y}$ ) may probably be absorbed by exciting the carriers to the higher energy band $E_{B Y}$ (shown as yellow shaded region in Fig. 3) or may be absorbed at the higher energy band edges of $E_{B X}$ (overlapping green and yellow shaded region of B) and $E_{A Y}$ (transparent green shaded region of A) due to their overlapping DOS, thus, occupying more carriers than usual. Simi- larly, the lower energy photons $\hbar \omega_{C X}$ generated by carrier capture in the $E_{C X}$ band most probably get absorbed either in $E_{B X}$ (green shaded region of B) or in $E_{A X}$ and $E_{A Y}$ (orange and green shaded regions of $\mathrm{A}$, respectively). However, the available DOS in $E_{B X}$ is quite large compared to $E_{A Y}$ (which has only the DOS tail in that energy region). Thus, most of the photons (with energy $\hbar \omega_{C X}$ ) get absorbed in B and few in A. Notice that regardless of a large available DOS in the $E_{A X}$ band, negligible carriers may reside in this band because the acquired energy of the carriers (either from $\hbar \omega_{C X}$ or from $\hbar \omega_{C Y}$ photon absorption) are larger or equal to the high energy band edge of $E_{A X}$. Therefore, the above discussion may imply that group B Qdashes subsequently become dominant and the peak lasing wavelength shifts to longer wavelengths due to reduction in the GS transition energy characterized by $E_{B 0}$. Increasing the inhomogeneity value (or cavity length) further may shift the dominant Qdash group from B to A in a similar manner as mentioned above and, hence, the peak lasing wavelength shifts to the red. This is a unique feature observed only in Qdash laser structures.

In summary, we have developed a model to calculate and analyze the spectral and dynamical characteristics of InAs/ InP Qdash lasers. The calculated lasing spectra from the model confirmed the observation of redshift as a function of cavity length. We correlated this observation to the inhomogeneity of the active region incorporating the DOS of Qdashes. Based on this correlation, we presented a qualitative energy band model on the redshift in the lasing spectra.

The work is supported by a grant from KAUST- University of Michigan's AEA 2010.

${ }^{1}$ F. Lelarge, B. Dagens, J. Renaudier, R. Brenot, A. Accard, F. van Dijk, D. Make, O. Le Gouezigou, J. G. Provost, F. Poingt, J. Landreau, O. Drisse, E. Derouin, B. Rousseau, F. Pommereau, and G. H. Duan, IEEE J. Sel. Top. Quantum Electron. 13, 111 (2007).

${ }^{2}$ J. Reithmaier, G. Eisenstein, and A. Forchel, Proc. IEEE 19, 1779 (2007). ${ }^{3}$ B. S. Ooi, H. S. Djie, Y. Wang, C. L. Tan, J. C. M. Hwang, X. M. Fang, J. M. Fastenau, W. K. Liu, G. T. Dang, and W. H. Chang, IEEE J. Sel. Top. Quantum Electron. 14, 1230 (2008).

${ }^{4}$ H. S. Djie, C. L. Tan, B. S. Ooi, J. C. M. Hwang, X. M. Fang, Y. Wu, J. M. Fastenau, W. K. Liu, G. T. Dang, and W. H. Chang, Appl. Phys. Lett. 91, 111116 (2007).

${ }^{5}$ C. L. Tan, H. S. Djie, Y. Wang, C. E. Dimas, V. Hongpinyo, Y. H. Ding, and B. S. Ooi, Appl. Phys. Lett. 93, 111101 (2008).

${ }^{6}$ C. L. Tan, H. S. Djie, Y. Wang, C. E. Dimas, V. Hongpinyo, Y. H. Ding, and B. S. Ooi, IEEE Photon. Technol. Lett. 21, 30 (2009).

${ }^{7}$ D. Zhou, R. Piron, M. Dontabactouny, E. Homeyer, O. Dehaese, T. Batte,

M. Gicquel, F. Grillot, K. Tavernier, J. Even, and S. Loualiche, Phys. Status Solidi C 10, 2217 (2009).

${ }^{8}$ R. Schwertberger, D. Gold, J. P. Reithmaier, and A. Forchel, IEEE Photon. Technol. Lett. 14, 735 (2002).

${ }^{9}$ M. Sugawara, K. Mukai, Y. Nakata, and H. Ishikawa, Phys. Rev. B 61 , 7595 (2000).

${ }^{10}$ M. Gioannini, IEEE J. Quantum Electron. 40, 364 (2004).

${ }^{11}$ Z. Mi and P. Bhattacharya, IEEE J. Quantum Electron. 42, 1224 (2006).

${ }^{12}$ J. H. Wei and K. S. Chan, J. Appl. Phys. 97, 123524 (2005).

${ }^{13}$ S. C. Heck, S. Osborne, S. B. Healy, E. P. O'Reilly, F. Lelarge, F. Poingt, O. L. Gouezigou, and A. Accard, IEEE J. Quantum Electron. 45, 1508 (2009)

${ }^{14}$ O. Qasaimeh, J. Lightwave Technol. 28, 1906 (2010).

${ }^{15}$ D. Hadass, A. Bilenca, R. Alizon, H. Dery, V. Mikhelashvili, G. Eisenstein, R. Schwertberger, A. Somers, J. P. Reithmaier, A. Forchel, M. Calligaro, S. Bansropun, and M. Karkowski, IEEE J. Sel. Top. Quantum Electron. 11, 1015 (2005).

${ }^{16}$ H. Dery and G. Eisenstein, IEEE J. Quantum Electron. 40, 1398 (2004). 